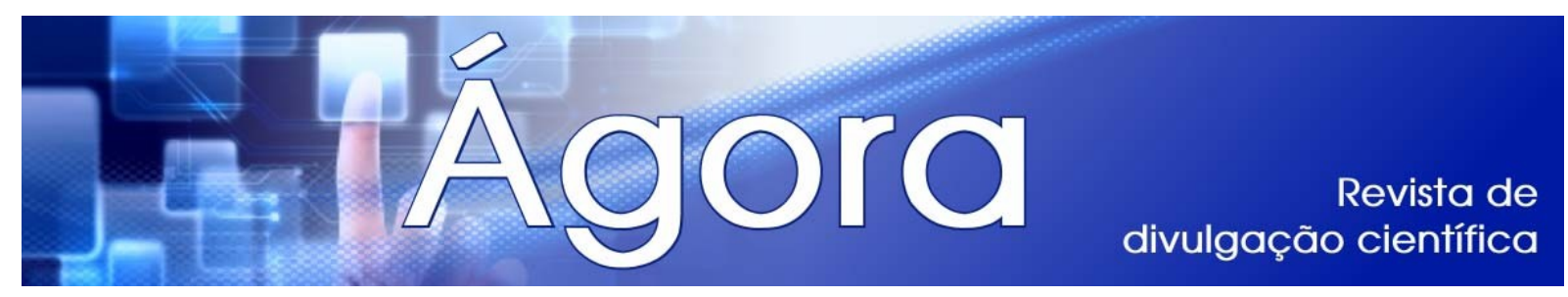

\title{
AVALIAR OS EFEITOS DA CORRENTE RUSSA NA REGIÃO GLÚTEA COMO COADJUVANTE NA ATIVIDADE FÍSICA COM FINALIDADE ESTÉTICA
}

\author{
Tailine Fernandes Ribas ${ }^{1}$ \\ Danila Soares Ritter ${ }^{2}$ \\ Jaqueline Sueli Horodeski ${ }^{3}$
}

RESUMO: Introdução $O$ cuidado com a aparência física, principalmente em mulheres, aumentou a procura por fisioterapeutas dermatofuncionais para tratamentos estéticos. Um glúteo firme, durinho e arredondado é o sonho de toda mulher. Com o passar dos anos, seja pela genética ou pelo sedentarismo, a região glútea tende a perder o tônus muscular. O tratamento denominado "corrente russa", técnica de eletroestimulação neuromuscular desenvolvida no final dos anos 70 para prover ganho de força em atletas, também tem sido utilizado, nos dias atuais, para fins estéticos. Este trabalho consistiu em uma análise dos efeitos da corrente russa aplicada na musculatura da região glútea de mulheres praticantes de atividades físicas, como agente acelerador da hipertrofia muscular. Metodologia: foram selecionadas oito mulheres saudáveis, praticantes de atividades físicas, com grau 5 de força muscular em região glútea, separadas em dois grupos iguais: Grupo A, formado por quatro mulheres submetidas a quinze sessões de corrente russa; Grupo $\mathrm{B}$, ou grupo controle, composto de mulheres não submetidas à corrente russa. Ambos os grupos foram avaliados pré e pós período das sessões de corrente russa, em termos de perimetria da região glútea, peso corporal, percentual de gordura corporal, Escala de Opinião (OENNING, 2002), além da opinião pessoal das participantes sobre os efeitos da corrente russa. Objetivos: avaliar os efeitos da eletroestimulação neuromuscular do tipo "corrente russa" na musculatura da região glútea, como coadjuvante na atividade física, com finalidade estética; verificar se a corrente russa provoca hipertrofia muscular em região glútea; comparar os resultados obtidos do grupo submetido à corrente russa com o grupo controle. Resultados: a análise dos resultados numéricos obtidos, apesar de favorável em relação ao grupo controle e em relação à avaliação inicial, não revelou dados significativos, provavelmente, devido ao tamanho reduzido das amostras $(p=0,61)$. Porém, na pesquisa de satisfação, a opinião pessoal das quatro componentes do grupo A foi unânime quanto à melhora da aparência da região glútea após o tratamento com corrente russa. Conclusão: foi possível concluir que houve melhora em termos de hipertrofia muscular em região glútea das participantes submetidas à corrente russa, assim como resultados positivos de satisfação pessoal das mesmas.

\footnotetext{
${ }^{1}$ Acadêmica do curso de Fisioterapia da Universidade do Contestado (UnC). E-mail: tay ribas@hotmail.com

2 Acadêmica do curso de Fisioterapia da Universidade do Contestado (UnC). E-mail: srdanila@hotmail.com

${ }^{3}$ Professora orientadora. Docente do curso de Fisioterapia da Universidade do Contestado (UnC). Email: jaqueline@unc.br
}

Ágora: R. Divulg. Cient., v. 18, n. 2, p. 53-63, dez. 2011 (ISSN 2237-9010) 
Palavras-chave: Dermatofuncional. Eletroestimulação neuromuscular. Corrente russa. Hipertrofia muscular. Glúteos.

ABSTRACT: Introduction the care with physical appearance, especially in women, has increased demand for aesthetic treatments performed by dermato-functional physiotherapists. A firm, solid and rounded glutes is the dream of every woman. Over the years, due to genetics and inactivity, the gluteal region tends to lose muscle tone. The treatment called "Russian current", a neuromuscular electrical stimulation technique, developed in the late '70s to provide strength gains in athletes, has also been used, nowadays, for aesthetic purposes. This study consisted of an analysis of the effects of Russian currenttreatment as an accelerating agent of muscular hypertrophy, applied to the muscles of gluteal region of women who practice usually physical activities. Methods: We selected eight women who had to be healthy, physically active individuals, with grade 5 of muscle strength in the gluteal region, and separated them into two groups: Group "A", formed by four women who were subjected to fifteen sessions of Russian current treatment; and Group B, also called control-group, composed of four women who were not subject to that treatment. Both groups were assessed before and after the period of fifteen sessions of Russian current in terms of gluteal region's girth, body weight, body fat percentage, Opinion Scale (Oenning, 2002), and the personal opinion of the participants about the effects of Russian current in their bodies. Objectives: evaluating the effects of neuromuscular electrical stimulation called Russian current in the muscles of gluteal region, as an adjunct in physical activity, with aesthetic purpose; verify if Russian current treatment causes muscle hypertrophy in the gluteal region; compare the results of the group undergoing Russian current treatment with the control-group. Results: The analysis of the numerical results obtained data revealed no significant improvement after the treatment, even though favorable compared to the controlgroup and initial evaluation. This was probably due to the small size of the samples $(p=0.61)$. However, the personal opinion of the four components of group $A$ at the satisfaction survey was unanimous as to the improvement of the appearance of the gluteal region after Russian current treatment. Conclusion: we concluded that there was an improvement in terms of muscle hypertrophy in the gluteal region of participants undergoing Russian current treatment, as well as positive results of personal satisfaction from them.

Keywords: Dermato-functional. Neuromuscular electrical stimulation. Russian current treatment. Muscle hypertrophy. Gluteal region.

\section{INTRODUÇÃO}

O padrão de estética feminina tem mudado através dos tempos. $\mathrm{Na}$ antiguidade, a obesidade era sinônimo de beleza feminina. Em meados do século XX, na década de 60, passou-se a buscar a magreza como modelo estético de 
beleza. A partir das décadas de 80 e 90, o padrão de beleza novamente começou a mudar, influenciado principalmente pela mídia, com o aparecimento de ícones da música e do cinema, como Madonna, Grace Jones, ou Demi Moore, que passaram a exibir seus corpos com a musculatura aparente e baixo percentual de gordura (GUEDES, 2005).

Os treinamentos físicos baseados em exercícios resistidos, que há algumas décadas eram atividade quase que exclusiva do público masculino, passaram a ser praticados por homens e mulheres na mesma proporção. Normalmente, com esses treinamentos, buscam-se resultados voltados à hipertrofia muscular, para realçar os contornos do corpo, obviamente com intenção estética, uma vez que a hipertrofia, por si só, não promove nenhum benefício funcional no corpo, visto que é apenas uma adaptação corporal à sobrecarga de pesos em exercícios resistidos (GUEDES, 2005).

Atualmente, no Brasil, uma das grandes preocupações estéticas do público feminino está relacionada à forma das regiões abdominal, glútea e de membros inferiores, o que provocou uma demanda por exercícios denominados popularmente como "ginástica localizada", que nada mais são do que exercícios focalizados no fortalecimento dos grupos musculares destas regiões.

O cuidado com a manutenção ou melhora da aparência física, por parte das mulheres, tem abrangido uma variedade cada vez maior de especialidades profissionais, seja na área médica, fitness ou fisioterapêutica. Uma das técnicas fisioterapêuticas mais utilizadas atualmente na área dermato funcional denomina-se "corrente russa", ou "estimulação russa". Esta técnica de eletroestimulação neuromuscular (EENM) tem proporcionado resultados satisfatórios em tratamentos estéticos, principalmente no que diz respeito a fortalecimento muscular.

Com o passar dos anos, a corrente russa tem sido cada vez mais aplicada em tratamentos com fins estéticos. Por sua vez, a região glútea é alvo constante da atenção das mulheres, no que diz respeito à manutenção da autoestima. Por causa disso, a prática de exercícios físicos resistidos, direcionados para a melhoria do aspecto estético, daquela região é amplamente disseminada. Acredita-se que a utilização da corrente russa associada à prática regular de exercícios físicos direcionados à musculatura glútea possa acelerar a hipertrofia muscular daquela 
região, atendendo ao interesse estético tão buscado pelas mulheres na atualidade, que se traduz, popularmente, pela aparência de um "bumbum mais empinado".

Os objetivos deste estudo concentraram-se em avaliar os efeitos estéticos da eletroestimulação neuromuscular do tipo "corrente russa", aplicada na musculatura da região glútea de mulheres saudáveis e praticantes regulares de atividades físicas, com enfoque no fortalecimento e hipertrofia desta musculatura; verificar se a corrente russa provoca hipertrofia muscular em região glútea, e comparar os resultados obtidos do grupo submetido à corrente russa com o grupo controle.

\section{MATERIAIS E MÉTODOS}

O universo da pesquisa foi definido pela escolha de mulheres praticantes de atividade física, e que possuíssem grau 5 de força muscular.

A partir desta delimitação, foram recrutadas 8 mulheres em uma academia de musculação do município de Rio Negro, Paraná, que foram separadas em dois grupos, com o mesmo número de componentes, sendo definidos como:

a) Grupo "A": mulheres praticantes de atividade física que foram submetidas à corrente russa;

b) Grupo "B" (grupo controle): mulheres praticantes de atividade física.

No intuito de obter maior confiabilidade nos resultados obtidos, uma vez que o trabalho envolveu pequenas amostras, foram definidos os seguintes critérios de inclusão:

-mulheres, que estivessem praticando regularmente atividade física, objetivando hipertrofia muscular em região glútea, há no mínimo três meses consecutivos;

-os componentes de ambos os grupos não poderiam alterar seu programa de treinamento e hábitos de vida no período da pesquisa;

-idade entre 20 e 41 anos;

-massa corpórea entre 55 e 65kg;

-possuir grau 5 de força muscular, segundo Torres (2006): "amplitude de movimento completa contra a ação da gravidade e com resistência máxima". 
Além destes critérios, todos os componentes tiveram que concordar e assinar o Termo de Consentimento Livre e Esclarecido (TCLE).

Os critérios de exclusão ficaram assim definidos:

-mulheres com restrições médicas à utilização da corrente russa;

-mulheres que não atendessem aos critérios de inclusão;

-pessoas do sexo masculino.

As sessões de fisioterapia foram realizadas na clínica-escola da Universidade do Contestado - Campus Mafra.

\section{RESULTADOS E DISCUSSÃO}

Para análise dos resultados, convém relembrar que a amostra foi composta por oito integrantes do sexo feminino, separadas em dois grupos de quatro componentes, sendo: Grupo A (pacientes submetidos à corrente russa) e Grupo B (grupo controle). Os dados foram analisados utilizando planilhas eletrônicas dos softwares Microsoft $\AA$ Excel $\AA$ e GraphPad Prism 6.01®, onde foram comparados os dados das pacientes dos dois grupos, e os resultados, plotados em gráficos. Os dados colhidos na pesquisa foram analisados em cinco subitens.

\section{ANÁLISE DA EVOLUÇÃO DA PERIMETRIA DE REGIÃO GLÚTEA}

Esta é a análise mais importante deste estudo, pois a perimetria de região glútea é o dado mensurável que melhor caracteriza a hipertrofia muscular naquela região.

O Gráfico 1 mostra um aumento médio de perimetria em região glútea da ordem de $1,2 \%$ para o grupo A (corrente russa), e de $0,4 \%$ para o grupo B (grupo controle), nos períodos inicial e final da pesquisa. 


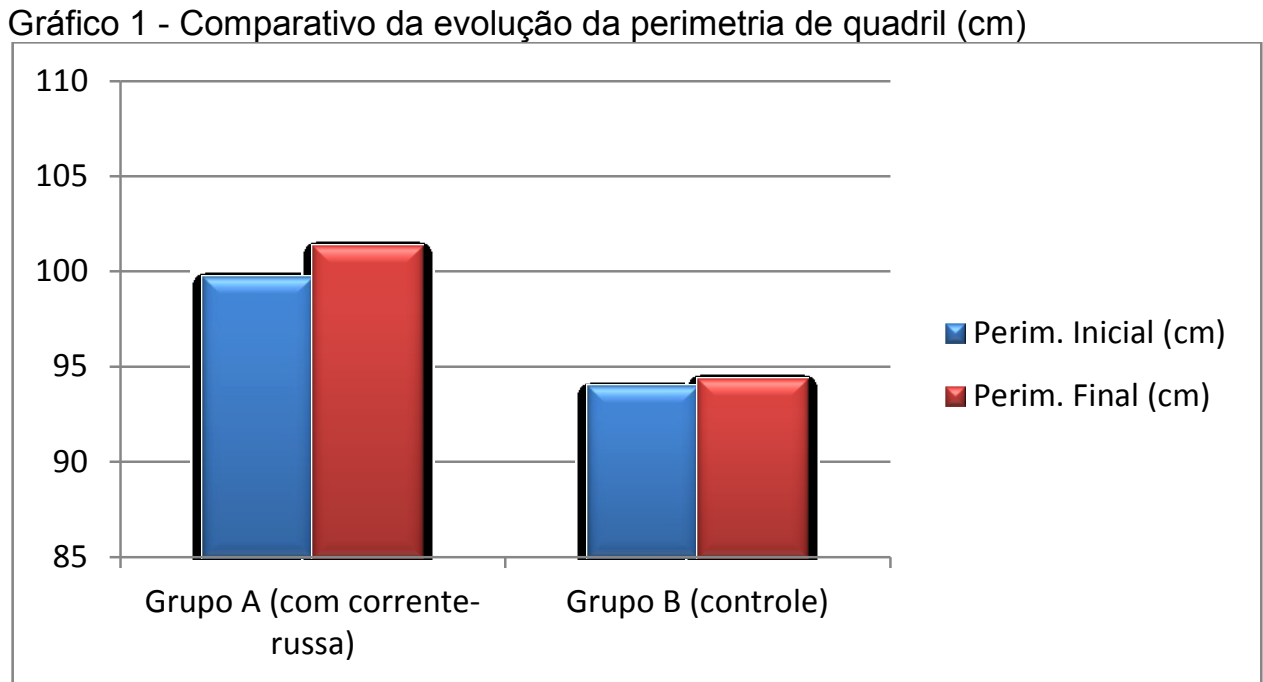

Fonte: Ribas; Ritter; Horodéski (2012)

A análise estatística dos resultados obtidos em evolução da perimetria foi realizada com base na comparação das hipóteses nula e alternativa, utilizando-se o teste "t" de Student para avaliação do resultado.

Hipótese nula (Ho): as aplicações de corrente russa não provocam aumento significativo da perimetria em região glútea.

Hipótese alternativa $(\mathrm{H} 1)$ : as aplicações de corrente russa provocam aumento significativo da perimetria em região glútea. Esta hipótese é considerada bilateral, pois a variação pode ocorrer nos dois sentidos.

Nível de significância ( $\alpha$ ) utilizado: $5 \%$. Graus de liberdade: 6

Gráfico 2 - Evolução da perimetria $(\mathrm{cm})$ de quadril do grupo A

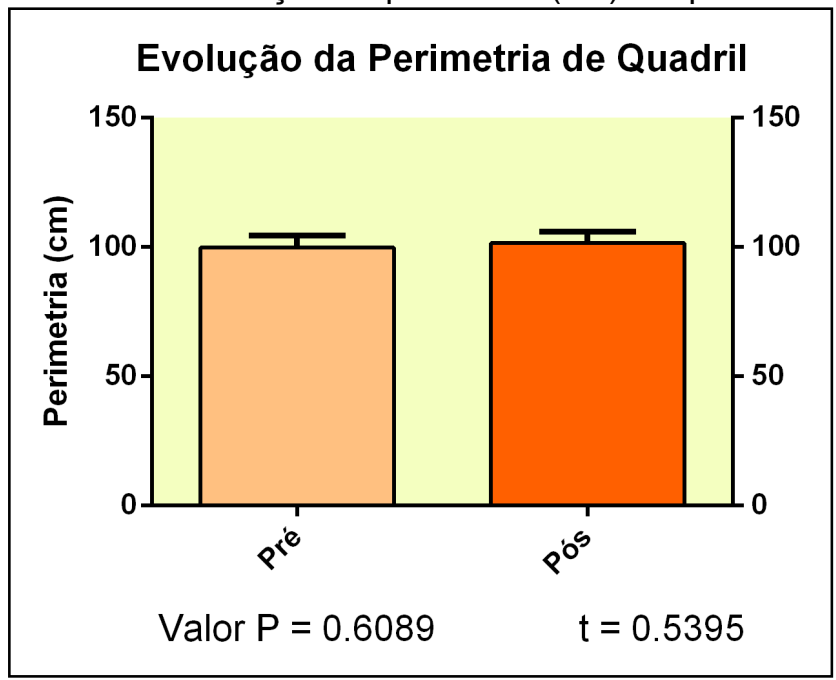

Fonte: Ribas; Ritter; Horodéski (2012) 
$O$ valor $P$ obtido de 0,6089 $(p<1)$, levando-se em consideração um nível de significância $\alpha$ (alfa) $=5 \%$, não nos permite descartar a hipótese nula com segurança (Gráfico 2).

Portanto, em termos estatísticos, o aumento de perimetria obtido após as sessões de corrente russa não é relevante, podendo ser atribuído ao acaso. Para maior confiabilidade dos resultados, seria indicado trabalhar com amostras maiores.

O valor de "t" de 0,5395, analisado para um nível de significância a de 5\%, possuindo a amostra um total de 6 graus de liberdade, está menor do que o valor de referência de tabela, que é de 2,45. Tal análise mostra que as médias de perimetrias iniciais e finais não possuem diferenças significativas, ou seja, estatisticamente, a corrente russa não proporcionou aumento de perimetria significativo.

A Tabela 1 contém os dados iniciais e finais de perimetria de região glútea do grupo $A$, submetido às sessões de corrente russa.

Tabela 1 - Dados de evolução da perimetria de região glútea do grupo A

\begin{tabular}{l|c|c}
\hline & Inicial & Final \\
\hline \multirow{2}{*}{ Perimetria quadril $(\mathrm{cm})$} & 95 & 97 \\
\cline { 2 - 3 } & 100,5 & 103 \\
\cline { 2 - 3 } & 106 & 107 \\
\cline { 2 - 3 } Média & 97,5 & 99 \\
\hline Variância & 99,8 & 101,4 \\
\hline Variância ponderada & 22,42 & 19,23 \\
\hline
\end{tabular}

Fonte: Ribas; Ritter; Horodéski (2012)

\section{ANÁLISE DA EVOLUÇÃO DO PESO MÉDIO}

A análise da evolução do peso médio das participantes dos dois grupos está demonstrada no Gráfico 3: 
Gráfico 3 - Evolução do peso $(\mathrm{kg})$ das participantes ao início e ao final da pesquisa

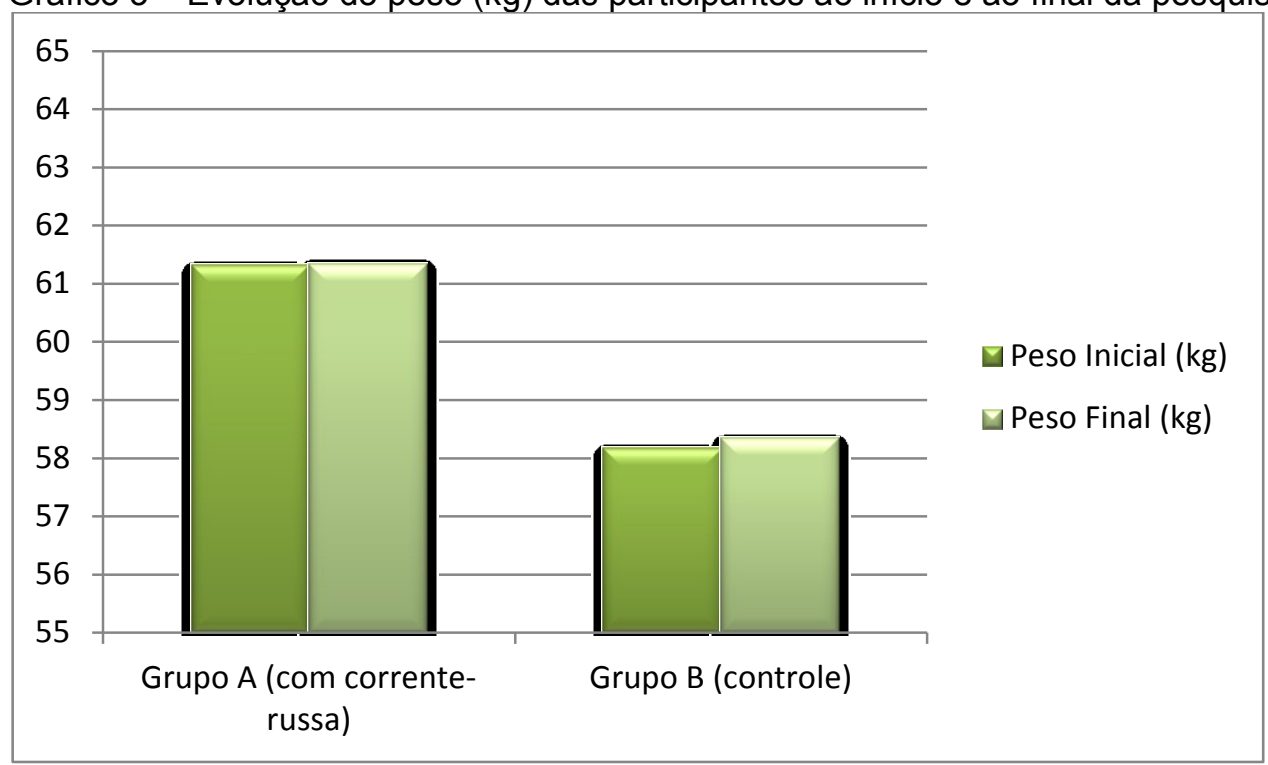

Fonte: Ribas; Ritter; Horodéski (2012)

Apesar de o grupo A apresentar menor variação de peso em relação ao grupo controle, o Gráfico 3 demonstra que a evolução do peso das participantes no período não mostrou alterações significativas, sendo sua análise irrelevante para o presente estudo, pois está associada a diversas variáveis.

\section{EVOLUÇÃO DO PERCENTUAL DE GORDURA (DOBRAS CUTÂNEAS)}

A evolução do percentual de gordura dos dois grupos segundo o protocolo de dobras cutâneas para mulheres (Siri-1961/Guedes-1994) foi realizada antes e após período das quinze sessões de corrente russa, estando demonstrada no Gráfico 4, abaixo:

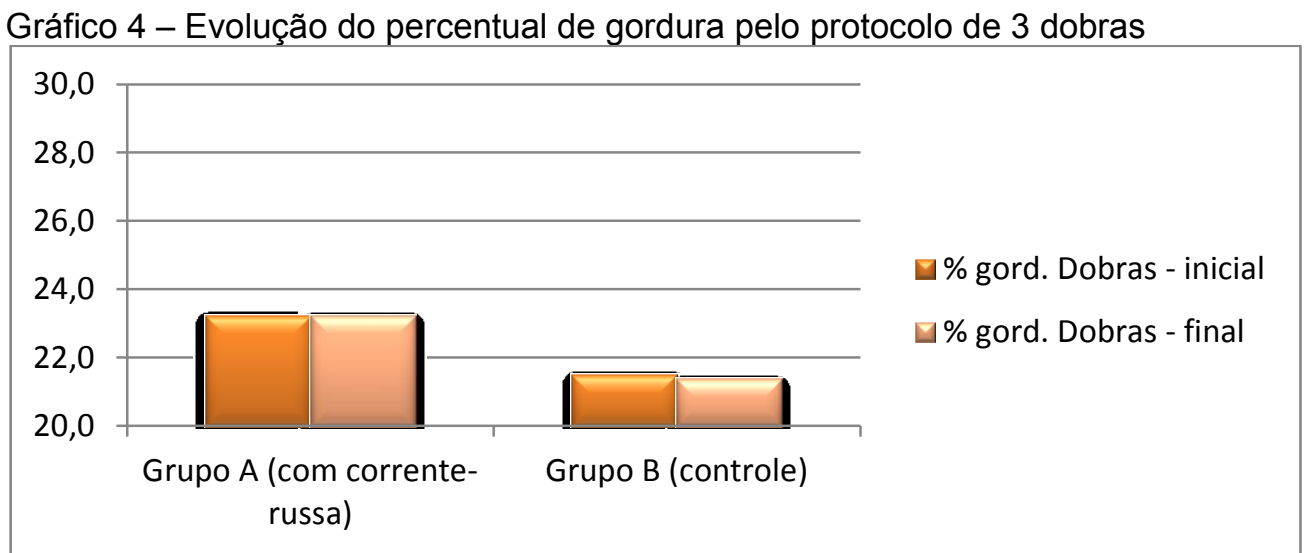

Fonte: Ribas; Ritter; Horodéski (2012)

Ágora: R. Divulg. Cient., v. 18, n. 2, p. 53-63, dez. 2011 (ISSN 2237-9010) 
O protocolo de 3 dobras demonstra leve redução do percentual de gordura dos dois grupos no período da pesquisa, que provavelmente está associada à prática usual de atividades físicas, por parte das participantes da pesquisa.

\section{EVOLUÇÃO DO PERCENTUAL DE GORDURA (BIOIMPEDÂNCIA)}

A evolução do percentual de gordura dos dois grupos segundo o medidor eletrônico de bioimpedância está demonstrada no Gráfico 5 . A variação pré e pós tratamento foi mínima, assim como pelo protocolo de dobras cutâneas.

Gráfico 5 - Evolução do percentual de gordura conforme aparelho de bioimpedância

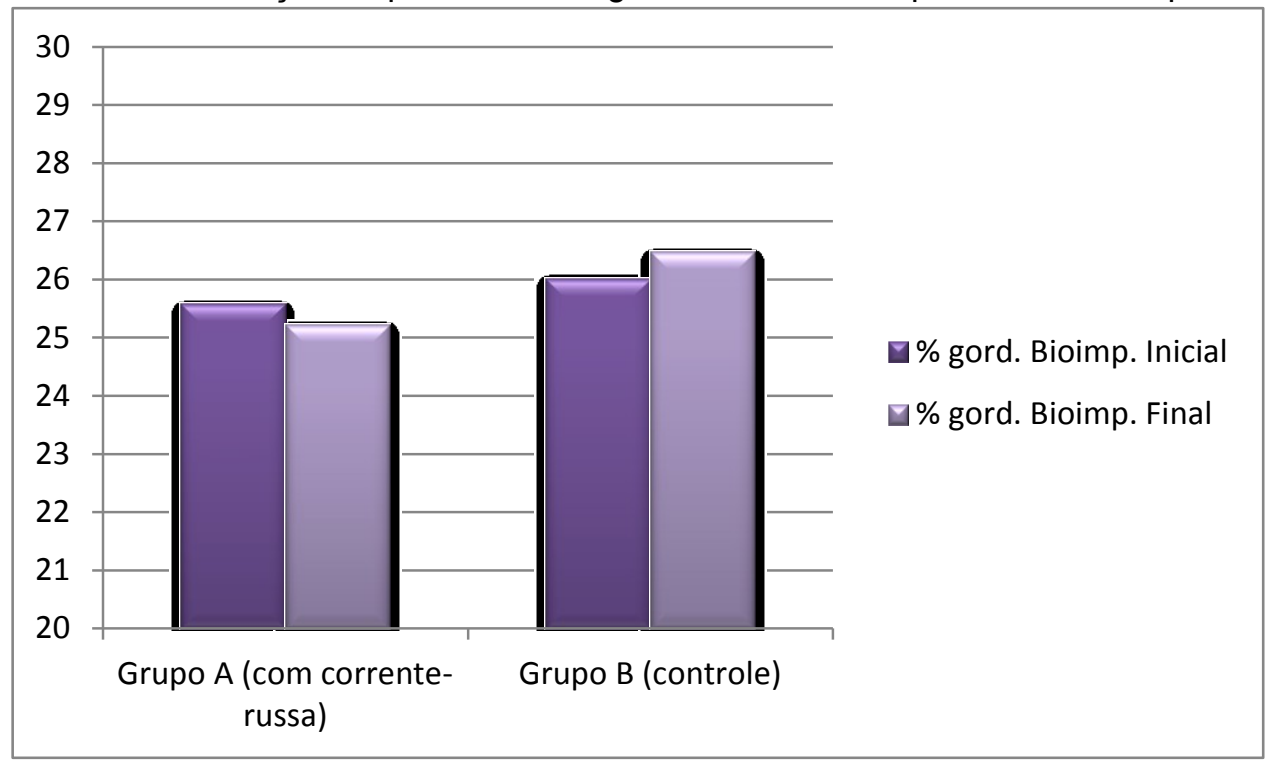

Fonte: Ribas; Ritter; Horodéski (2012)

\section{ANÁLISE DE OPINIÃO DAS PARTICIPANTES}

A análise da opinião pessoal das participantes da pesquisa (Grupo A), para avaliar o nível de satisfação em relação ao tratamento, foi realizada após o tratamento com corrente russa, conforme a Escala de Opinião (OENNING, 2002). Foi formulada a questão: "Qual o seu nível de satisfação após o tratamento com corrente russa?", havendo as opções de resposta: "plenamente satisfeita", "satisfeita", "parcialmente satisfeita", "insatisfeita". Desta pesquisa, obteve-se o seguinte resultado: 
Avaliar os efeitos da corrente russa na região glútea como coadjuvante na atividade física com finalidade estética

Tabela 2 - Pesquisa de satisfação (Grupo A)

Escala de Opinião (Oenning, 2002)

"Qual o seu nível de satisfação após o tratamento com corrente russa?"

\begin{tabular}{l|c}
\hline Plenamente satisfeita & 4 \\
\hline Satisfeita & 0 \\
\hline Parcialmente satisfeita & 0 \\
\hline Insatisfeita & 0
\end{tabular}

Fonte: Oenning, 2002 (adaptada por Ribas; Ritter; Horodéski, 2012)

A Tabela 2 demonstra que 100\% das participantes submetidas ao tratamento com corrente russa sentiram-se plenamente satisfeitas após a última sessão.

\section{CONCLUSÃO}

Os resultados obtidos, quanto à evolução da perimetria de quadril do grupo submetido à corrente russa associada aos exercícios físicos, assim como a comparação dos resultados dos Grupos A e B, apesar de favoráveis para a corrente russa em números absolutos, e em concordância com os estudos de outros pesquisadores, não podem ser considerados estatisticamente confiáveis, devido ao tamanho reduzido da amostra $(p=0,6089)$.

No entanto, a ferramenta estatística não descarta de todo a hipótese alternativa, o que ficou confirmado através da unanimidade das participantes do Grupo A quanto à melhoria da aparência da região glútea após o tratamento com corrente russa, segundo informações colhidas.

Desta forma, é possível considerar que é benéfica a utilização da corrente russa associada à prática de exercícios físicos, em termos de hipertrofia muscular.

\section{REFERÊNCIAS}

BORGES, Fábio dos S. et al. Parâmetros de modulação na eletroestimulação neuromuscular utilizando corrente russa: Parte 2. Revista Fisioterapia Ser, a. 2, n. 2, abr./jun. 2007. Disponível em: <http://www.proffabioborges.com.br/artigos/ parametros_modulacao_eenm_corrente_russa_parte2.pdf>. Acesso em 10 abr. 2012.

GUEDES, Dilmar P. Musculação: estética e saúde feminina. 2. ed. São Paulo: Phorte, 2005.

GUIRRO, Elaine; GUIRRO, Rinaldo. Fisioterapia Dermato-Funcional:

fundamentos, recursos, patologias. 3. ed. Barueri: Manole, 2004. 
NAHAS, M.V. Atividade física, saúde e qualidade de vida: conceitos e sugestões para um estilo de vida ativo. 4 ed. Londrina: Midiograf, 2006.

NELSON, Roger M.; HAYES, Karen W.; CURRIER, Dean P. Eletroterapia clínica. 3. ed. São Paulo: Manole, 2003.

RAMOS, A. T. Treinamento de força na atualidade. Rio de janeiro: Sprint, 2002.

REMOR, B.M.; ROSAS, R.F. Corrente russa versus exercício resistido na avaliação do fortalecimento muscular em idosos institucionalizados. $2008.7 \mathrm{f}$. Trabalho de Conclusão de Curso (Graduação em Fisioterapia) - UNISUL, Tubarão, 2008. Disponível em: <http://www.fisio-tb.unisul.br/Tccs/08b/bruno/ARTIGO.pdf>. Acesso em: 31 out. 2012.

ROBERGS, R. A.; ROBERTS, S. O. Princípios fundamentais de fisiologia do exercício para aptidão, desempenho e saúde. São Paulo: Phorte, 2002.

ROBERTO, Alexander E. Eletroestimulação: o exercício do futuro. São Paulo: Phorte, 2006.

ROBINSON, Andrew J.; SNYDER-MACKLER, Lyn. Eletrofisiologia clínica. 2. ed. Porto Alegre; Artmed, 2002.

SANTOS, A.N.; NICOLAU, R.A.; PACHECO, M.T.T. Efeito da Terapia com Estimulação Russa em Músculo Reto Abdominal Observado Através de UltraSonografia. In: ENCONTRO LATINO AMERICANO DE INICIAÇÃO CIENTÍFICA SANTOS, IX, 2005, João Pessoa. Anais eletrônicos... João Pessoa: UNIVAP, 2005. p. 1413-1416.

SILVA, R.T.; FILHO, H.T. Efeitos do treinamento de força associado ao uso de eletroestimulação neuromuscular em membros inferiores: Revista Digital EFDeportes.com, 2009. Disponível em: <http://www.efdeportes.com/efd129/ treinamento-de-forca-associado-ao-uso-de-eletroestimulacao-neuromuscular.htm>. Acesso em: 02 nov. 2012.

TRAVELL, Janet G., SIMONS, David G. Myofascial pain and dysfunction. Philadelphia: Lippincott Williams \& Wilkins, 1997.

UCHIDA, M.C. et al. Manual de musculação: uma abordagem teórico-prática do treinamento de força. 3. ed. São Paulo: Phorte, 2005. 